\title{
The Glymphatic System: A Potential Pathophysiological Focus for Perioperative Neurocognitive Disorder
}

\author{
Zhonghua Hu, Fan Zhang, Qin Liao and Wen Ouyang* \\ Department of Anesthesiology, the Third Xiangya Hospital, Central South University, \\ No. 138 Tongzipo Road, Yuelu District, Changsha, Hunan, China
}

\begin{abstract}
Perioperative neurocognitive disorder is a decline in cognitive function of patients after anesthesia and surgery. It seems to be a comprehensive effect of the combination of psychological state before the operation, neurotoxicity of narcotic drugs, inflammation caused by the operation, and sleep deprivation after surgery. The glymphatic pathway is a newly discovered system that clears metabolic waste from the brain. The clearing efficiency of the glymphatic pathway can be influenced by sleep deprivation, some narcotic drugs (like dexmedetomidine and ketamine), and neuroinflammation. We hypothesize that the glymphatic system may play a pivotal role in the occurrence and development of perioperative neurocognitive disorder.
\end{abstract}

\section{Introduction}

Perioperative neurocognitive disorder (PND) is a decline in cognitive function of patients after anesthesia and surgery, characterized by impairment of executive function, memory, attention, language comprehension, and other cognitive domains. PND can be present as delirium for days or for more than 12 months, and in a minority of patients the impairment can continue or reemerge. ${ }^{1}$ Previously, it was called postoperative cognitive dysfunction (commonly referred to as POCD) but a change to PND was recommended in 20182; this change better aligns PNDs with the phenotypically similar neurocognitive diagnoses listed in the Diagnostic and Statistical Manual of Mental Disorders, version 5. Unified and effective approaches for the prevention and treatment of PND in clinical practice are lacking because its exact mechanism has not been elucidated completely.

The glymphatic pathway was first described in $2012 .{ }^{3}$ It is a system that clears waste, including amyloid- $\beta(a \beta)$ and tau protein, from the brain. It also serves as a glia-lymph system for waste clearance in the brain that differs from the cerebrospinal fluid

Keywords: Perioperative neurocognitive disorder; Glymphatic pathway; Mechanism; Neuroinflammation; Anesthesia.

Abbreviations: AQP-4, aquaporin-4; CNS, central nervous system; CSF, cerebrospinal fluid; HMGB1, high molecular group box 1; IL, interleukin; PND, perioperative neurocognitive disorder; POCD, postoperative cognitive dysfunction; TNF, tumor necrosis factor.

Received: June 10, 2020; Revised: July 08, 2020; Accepted: July 13, 2020

*Correspondence to: Wen Ouyang, Department of Anesthesiology, the Third Xiangya Hospital, Central South University, 138 Tongzipo Road, Yuelu District, Changsha, Hunan 410013, China. Tel: +86-13873170415, Fax: +86-731-88618679, E-mail: ouyangwen133@vip.sina.com

How to cite this article: Hu Z, Zhang F, Liao Q, Ouyang W. The Glymphatic System A Potential Pathophysiological Focus for Perioperative Neurocognitive Disorder. Ex ploratory Research and Hypothesis in Medicine 2020;000(000):000-000. doi: 10.14218/ERHM.2020.00041.
(CSF) system. Since its discovery, more and more researchers have focused on it, finding that the glymphatic function may be involved in neurodegenerative diseases and neurological recovery after stroke or traumatic brain injury. ${ }^{4}$ Decreased glymphatic influx has been observed secondary to subarachnoid hemorrhage, acute ischemia, and multiple microinfarcts.

Hypothesis: Glymphatic System May Play A Pivotal Role in PND

Despite the consensus on the nomenclature and diagnostic criteria of PND, whether anesthesia and surgery can be considered as etiologies is still controversial. Given the particularity of the glymphatic system and the complexity of possible changes after anesthesia and surgery, we herein speculate that the glymphatic system may play a pivotal role in the occurrence and development of PND. New ideas for clinical prevention and treatment of PND should consider the changes in the glymphatic pathway after anesthesia and surgery.

Evaluation of the Hypothesis

Cognitive changes after surgery have been described in one form or another for more than 50 years, initially as a complication after cardiac surgery. Many later studies found that some patients undergoing non-cardiac surgery also suffered cognitive decline, although the incidence after non-cardiac surgery was lower. ${ }^{5} \mathrm{~Pa}-$ tients, especially elderly patients, suffered cognitive decline after many kinds of surgery, but because of differences in assessment tools and methods, the reported incidence of POCD varied greatly. Following the 2018 recommended change to PND, when the diagnosis process and method were given, ${ }^{2}$ Daiello et al. ${ }^{6}$ found that 
Table 1. Possible risk factors of perioperative neurocognitive disorder ${ }^{5}$

\begin{tabular}{|c|c|}
\hline Factor classification & Possible risk factors \\
\hline \multirow[t]{3}{*}{ Anesthetic factors } & Neurotoxicity of narcotic drugs \\
\hline & Anesthetic method \\
\hline & Perioperative anesthesia management \\
\hline \multirow[t]{5}{*}{ Surgical factors } & Type of surgery: major surgery \\
\hline & Length of surgery \\
\hline & Postoperative infection and other complications \\
\hline & Pain \\
\hline & Length of hospitalization \\
\hline \multirow[t]{6}{*}{ Patient factors } & Advanced age \\
\hline & Apolipoprotein E gene \\
\hline & Lower education level \\
\hline & Nutritional status \\
\hline & Perioperative psychological factors \\
\hline & Effects of other diseases (stroke/cardiovascular disease) \\
\hline
\end{tabular}

postoperative delirium and postoperative cognitive dysfunction may be distinct manifestations of perioperative neurocognitive deficits.

The possible risk factors of PND are shown in Table $1 .^{5}$ The main pathogenic processes of PND are neurotoxicity of narcotic drugs, postoperative inflammatory response (neuroinflammation), and dysregulation of neurotransmitters and comprehensive function. ${ }^{7,8}$ Investigations into PND have been focused excessively on the neurotoxicity of narcotic drugs. Anesthesia-related factors, including the type of anesthetic method (general vs. regional) and intraoperative pathophysiological changes (hypotension and hypoxemia), are not considered pathophysiological factors of PND. ${ }^{9}$ Many details, such as patients' psychological state before their operations, their expectations, the effectiveness of surgery, surgical complications, and the surgery's psychological impact on patients were overlooked. The human body is very complex, and preoperative anxiety has been associated with an increased risk of PND. ${ }^{10}$ Pain can affect patients' quality of life and cognitive level, and, similarly, sleep deprivation is associated with PND. ${ }^{11}$ To date, PND has been considered as a cognitive decline after anesthesia and surgery, in which something must have changed in the patients' brains. However, while the exact change is not known, postoperative neuroinflammation may play an important role.

Surgery means tissue destruction, upon which the damaged tissues can activate the peripheral immune system; meanwhile, high molecular group Box 1 protein (HMGB1) is passively released from traumatized tissue. The HMGB1 can bind to pattern recognition receptors on bone marrow-derived monocytes to induce translocation of the transcription factor nuclear factor (NF) $-\kappa B$ into the nucleus, which can up-regulate the transcription and translation of proinflammatory cytokines, ${ }^{12}$ such as interleukin (IL)-1, IL-6, and tumor necrosis factor (TNF)- $\alpha$. The peripheral cytokines can stimulate microglia through the vagus nerve to produce proinflammatory cytokines in the central nervous system $(\mathrm{CNS})^{13}$; meanwhile, the cytokines can increase permeability of the blood-brain barrier, which allows cytokines and potential neurotoxins to penetrate into the CNS. ${ }^{7}$ The cytokines and neurotoxins also can activate the microglia and trigger neuroinflammation, which cause PND by inter- fering with long-term potentiation to impair synaptic plasticity. ${ }^{8}$ Although neuroinflammation seems to be the main mechanism of PND, we still do not know why some patients get PND, while others do not, and young people often have surgery with significant amounts of tissue damage yet rarely develop PND. Because the pathophysiological mechanism is not clear, we have no effective pathway to prevention and therapy of PND. Clinical research has found that dexmedetomidine ${ }^{14}$ and ketamine ${ }^{15}$ may confer better postoperative neurocognitive function after anesthesia and surgery, but the exact mechanism is not yet known.

The glymphatic pathway may play a pivotal role in the occurrence and development of PND. Metabolic waste removal from the CNS is essential for maintaining brain homeostasis and normal function. The traditional view is that the clearance of metabolic waste from the brain mainly depends on two routes: through the blood-brain barrier into the blood circulation, and through diffusion into the CSF circulation. Affected by the molecular weight of metabolites, both routes have difficulty in effectively removing the metabolites produced by brain parenchyma. How to metabolize the metabolites in the brain quickly and effectively has been a question in the neuroscience field. The discovery of the brain glial lymphoid pathway may provide a powerful answer to these questions. The glymphatic pathway mainly includes a periarterial CSF inflow pathway, an outflow channel of perivenous tissue fluid and aquaporin-4 (AQP-4) on astrocytes connecting the above two channels. ${ }^{16}$ It plays an important role in the clearance of brain metabolites, but the clearance rate of metabolites with different molecular weights varies and the glymphatic pathway is influenced by many factors, such as drugs, sleep, and neuroinflammation. ${ }^{17,18}$

The deposition of $\mathrm{a} \beta$ protein, tau protein and other toxic proteins is an important cause of Alzheimer's disease. The radiation (marker of isotope clearance) of a $\beta$ protein in the whole brain was found to significantly increase after AQP-4 was knocked out by gene knockout technology, ${ }^{3}$ indicating that the glymphatic pathway involved in AQP-4 could effectively remove a $\beta$ protein. Using a similar approach, the researchers found that the glymphatic pathway was effective in removing tau protein and lactate. ${ }^{19}$ This series of studies demonstrated that the brain glymphatic system 
is involved in the pathogenesis of CNS diseases, especially some degenerative diseases. ${ }^{20}$ On this basis, the latest research has found that sleep can effectively increase the clearance of $a \beta$ protein. ${ }^{17}$ Its mechanism is to increase the flow of CSF and accelerate the transport and clearance of a $\beta$ protein by affecting the glymphatic pathway and increasing the interstitial space. These studies may partly explain why sleep deprivation after surgery can affect cognitive function. Moreover, a clinical study found that the effect of one night's sleep deprivation on the brain's a $\beta$ burden resulted in a higher a $\beta$ accumulation than with chronic sleep deprivation. ${ }^{21}$ We need to pay more attention to the effect of sleep deprivation on patients undergoing surgery, especially elderly patients. ${ }^{22}$

Similar to sleep, ketamine/methylthiazide can enhance the clearance of $\mathrm{a} \beta$ protein by the glymphatic pathway. Benveniste et al. ${ }^{23}$ found that glymphatic transport was enhanced by $32 \%$ in rats anesthetized with dexmedetomidine plus low-dose isoflurane, which shows that anesthetics can also affect the transport of the glymphatic pathway. The mechanism may not be the unconscious state caused by anesthetics but may be the repression of norepinephrine or another release. These studies can explain why dexmedetomidine and ketamine can confer better postoperative neurocognitive function after anesthesia and surgery. Sleep deprivation and pain caused by surgery can influence the clearance efficiency of the glymphatic pathway, causing patients' cognition to decline. The influence of neuroinflammation after surgery should also be considered.

As mentioned, AQP-4 connects the two channels and transports metabolic waste to the CSF. However, recent research has found that the human brain contains meningeal lymphatics, which can regulate the CNS lymphatic drainage and neuroinflammation. ${ }^{24}$ The meningeal lymphatics govern the inflammatory processes and immune surveillance of the CNS. The discovery of meningeal lymphatics not only provides the tissue basis for brain immunity but also provides theoretical support for the new pathway of metabolite excretion: that is, some metabolites may enter the peripheral lymphatic system directly through meningeal lymphatics after passing through the glymphatic pathway. We know the glymphatic system has a relation to meningeal lymphatics, and the outflow channel of the glymphatic system interlinks the meningeal lymphatic vessel. ${ }^{25}$ The glymphatic pathway may involve and influence neuroinflammation through meningeal lymphatics. Therefore, the neuroinflammation caused by surgery also relates to the glymphatic pathway, as we know neuroinflammation is an important mechanism of PND.

As mentioned above, neuroinflammation plays an important role in the development of PND and synaptic plasticity is the key mechanism, but this makes it hard to explain why young people rarely develop PND and postoperative delirium, which is acute after surgery. The role of synaptic plasticity is long-term, but it should take a long time to work. There are likely to be other mechanisms. Astrocytes are connected to the vascular wall through AQP-4 to occupy a crucial position in the blood-brain barrier and also astrocytes are connected with the synapses of neurons to communicate and transmit information. ${ }^{26}$ The structure and function of astrocytes are associated with aging. ${ }^{16}$ Increased brain barrier permeability and activated microglia after surgery may work on astrocytes through endoplasmic reticulum stress and Th1 cells ${ }^{7}$ which can affect the function of AQP-4 and the glymphatic system. The proinflammatory cytokines can also act directly on AQP-4. The rapid accumulation of metabolites in the brain can cause acute and chronic changes in brain function, ${ }^{27}$ which may cause PND. The interaction mechanisms between peripheral inflammation, microglia and the astrocyte need more research. Narcotic drugs, pain and sleep deprivation can affect the glymphatic system themselves, although the underlying mechanisms are still poorly understood.

\section{Future direction}

PND seems to be a comprehensive effect of anesthesia, surgery, sleep deprivation and pain related to an operation, and the development of PND likely involves a complicated interaction between patient- and procedure-related variables. No single pathophysiological process has garnered universal support as a single causative factor for PND but it must have a single pathophysiological process-it is just that we do not know it yet. From the perspective of current basic and clinical research, the single pathophysiological process may be the glymphatic system, because the glymphatic system has a relation to almost all the variables caused by anesthesia and surgery, such as pain, narcotic drugs, sleep deprivation and inflammation but more research is needed.

\section{Conclusions}

In view of the above analysis, although the exact pathophysiological mechanism of PND is still unclear, we believe that the glymphatic system may play a pivotal role in the occurrence and development of PND. Next, we need to systematically research the changes in the glymphatic system after anesthesia and surgery to provide new ideas for prevention and treatment of PND.

\section{Acknowledgments}

None.

\section{Funding}

The work performed in our laboratory was supported by a grant from the Science and Technology Program of Hunan Province, China (No. 2017SK50130).

\section{Conflict of interest}

No conflicting interest exists with this publication.

Author contributions

Draft writing (ZH, FZ), manuscript revision (QL, WO).

\section{References}

[1] Eckenhoff RG, Maze M, Xie ZC, Culley DJ, Goodlin SJ, Zuo ZY, et al. Perioperative Neurocognitive Disorder State of the Preclinical Science. Anesthesiology 2020;132(1):55-68. doi:10.1097/ aln.0000000000002956.

[2] Evered L, Silbert B, Knopman DS, Scott DA, DeKosky ST, Rasmussen LS, et al. Recommendations for the nomenclature of cognitive change associated with anaesthesia and surgery-2018. Br J Anaesth 2018;121(5):1005-1012. doi:10.1016/j.bja.2017.11.087. 
[3] Iliff JJ, Wang MH, Liao YH, Plogg BA, Peng WG, Gundersen GA, et al. A paravascular pathway facilitates CSF flow through the brain parenchyma and the clearance of interstitial solutes, including amyloid $\beta$. Sci Transl Med 2012;4(147):147ra111. doi:10.1126/scitransImed.3003748.

[4] Plog BA, Nedergaard M. The Glymphatic System in Central Nervous System Health and Disease: Past, Present, and Future. Annu Rev Pathol 2018;13:379-394. doi:10.1146/annurev-pathol-051217-111018.

[5] Green CM, Schaffer SD. Postoperative cognitive dysfunction in noncardiac surgery: A review. Trends Anaesth Crit Care 2019;24:40-48. doi:10.1016/j.tacc.2018.08.003.

[6] Daiello LA, Racine AM, Gou RY, Marcantonio ER, Xie ZC, Kunze LJ, et al. Postoperative Delirium and Postoperative Cognitive Dysfunction: Overlap and Divergence. Anesthesiology 2019;131(3):477-491. doi:10.1097/aln.0000000000002729.

[7] Lin XY, Chen YR, Zhang P, Chen G, Zhou YF, Yu X. The potential mechanism of postoperative cognitive dysfunction in older people. Exp Gerontol 2020;130:110791. doi:10.1016/j.exger.2019.110791.

[8] Subramaniyan S, Terrando N. Neuroinflammation and Perioperative Neurocognitive Disorders. Anesth Analg 2019;128(4):781-788. doi:10.1213/ANE.0000000000004053.

[9] Moller JT, Cluitmans P, Rasmussen LS, Houx P, Rasmussen H, Canet J, et al. Long-term postoperative cognitive dysfunction in the elderly: ISPOCD1 study. ISPOCD investigators. International Study of PostOperative Cognitive Dysfunction. Lancet 1998;351(9106):857-861. doi:10.1016/s0140-6736(97)07382-0.

[10] Du J, Plas M, Absalom AR, van Leeuwen BL, de Bock GH. The association of preoperative anxiety and depression with neurocognitive disorder following oncological surgery. J Surg Oncol 2020;121(4):676687. doi:10.1002/jso.25836.

[11] Wang $Y$, Shen $X$. Postoperative delirium in the elderly: the potential neuropathogenesis. Aging Clin Exp Res 2018;30(11):1287-1295. doi:10.1007/s40520-018-1008-8.

[12] Saxena S, Lai IK, Li R, Maze M. Neuroinflammation is a putative target for the prevention and treatment of perioperative neurocognitive disorders. Br Med Bull 2019;130(1):125-135. doi:10.1093/bmb/ Idz010.

[13] Safavynia SA, Goldstein PA. The Role of Neuroinflammation in Postoperative Cognitive Dysfunction: Moving From Hypothesis to Treatment. Front Psychiatry 2019;9:752. doi:10.3389/fpsyt.2018.00752.

[14] Yang W, Kong LS, Zhu XX, Wang RX, Liu Y, Chen LR. Effect of dexmedetomidine on postoperative cognitive dysfunction and inflammation in patients after general anaesthesia: A PRISMA-compliant systematic review and meta-analysis. Medicine 2019;98(18):e15383. doi:10.1097/MD.0000000000015383.

[15] Hovaguimian F, Tschopp C, Beck-Schimmer B, Puhan M. Intraoperative ketamine administration to prevent delirium or postopera- tive cognitive dysfunction: A systematic review and meta-analysis. Acta Anaesthesiol Scand 2018;62(9):1182-1193. doi:10.1111/ aas.13168.

[16] Benveniste H, Liu X, Koundal S, Sanggaard S, Lee H, Wardlaw J. The Glymphatic System and Waste Clearance with Brain Aging: A Review. Gerontology 2019;65(2):106-119. doi:10.1159/000490349.

[17] Mendelsohn AR, Larrick JW. Sleep facilitates clearance of metabolites from the brain: glymphatic function in aging and neurodegenerative diseases. Rejuvenation Res 2013;16(6):518-523. doi:10.1089/ rej.2013.1530.

[18] Lilius TO, Blomqvist K, Hauglund NL, Liu G, Staeger FF, Baerentzen S et al. Dexmedetomidine enhances glymphatic brain delivery of intrathecally administered drugs. J Control Release 2019;304:29-38. doi:10.1016/j.jconrel.2019.05.005.

[19] Lundgaard I, Lu ML, Yang E, Peng W, Mestre H, Hitomi E, et al. Glymphatic clearance controls state-dependent changes in brain lactate concentration. J Cereb Blood Flow Metab 2017;37(6):2112-2124. do i: $10.1177 / 0271678 \times 16661202$.

[20] Rasmussen MK, Mestre H, Nedergaard M. The glymphatic pathway in neurological disorders. The Lancet Neurology 2018;17(11):10161024. doi:10.1016/s1474-4422(18)30318-1.

[21] Shokri-Kojori E, Wang GJ, Wiers CE, Demiral SB, Guo M, Kim SW, et al. $\beta$-Amyloid accumulation in the human brain after one night of sleep deprivation. PNAS 2018;115(17):4483-4488. doi:10.1073/ pnas.1721694115.

[22] Bah TM, Goodman, Jlliff JJ. Sleep as a Therapeutic Target in the Aging Brain. Neurotherapeutics 2019;16(3):554-568. doi:10.1007/s13311019-00769-6.

[23] Benveniste $H$, Heerdt PM, Fontes $M$, Rothman DL, Volkow ND. Glymphatic System Function in Relation to Anesthesia and Sleep States. Anesth Analg 2019;128(4):747-758. doi:10.1213/ ANE.0000000000004069.

[24] Louveau A, Smirnov I, Keyes TJ, Eccles JD, Rouhani SJ, Peske JD, et al. Structural and functional features of central nervous system lymphatic vessels. Nature 2015;523(7560):337-341. doi:10.1038/nature14432.

[25] Louveau A, Plog BA, Antila S, Alitalo K, Nedergaard M, Kipnis J. Understanding the functions and relationships of the glymphatic system and meningeal lymphatics. J Clin Invest 2017;127(9):3210-3219. doi:10.1172/jci90603.

[26] Kim SK, Nabekura J, Koizumi S. Astrocyte-mediated synapse remodeling in the pathological brain. Glia 2017;65(11):1719-1727. doi:10.1002/glia.23169.

[27] Boland B, Yu WH, Corti O, Mollereau B, Henriques A, Bezard E, et al. Promoting the clearance of neurotoxic proteins in neurodegenerative disorders of ageing. Nat Rev Drug Discov 2018;17(9):660-688. doi:10.1038/nrd.2018.109. 